\title{
LA CULTURA COMO UN DETERMINANTE DE LIDERAZGO EMPRESARIAL EN CIUDAD CUAUHTÉMOC, CHIHUAHUA
}

\author{
Ema Cristina Gutiérrez Enríquez*, Karla Isabel Gutiérrez Enríquez
}

\begin{abstract}
Gutiérrez-Enriquez E.C., Gutiérrez-Enriquez K.I. La cultura como un determinante de liderazgo empresarial en Ciudad Cuauhtémoc, Chihuahua. Hitos de Ciencias Económico Administrativas 2014;20 (58): 103-111.
\end{abstract}

\section{RESUMEN}

Objetivo: Establecer las diferencias culturales en liderazgo empresarial, entre los empresarios de Ciudad Cuauhtémoc, Chihuahua.

Material y método: El estudio se llevó a cabo en ciudad Cuauhtémoc, Chihuahua, la información personal de cada uno de los empresarios, se obtuvo mediante entrevistas basadas en la historia de vida empresarial de doce empresarios, hombres de la región de Cuauhtémoc, siete de ellos fueron empresarios mestizos y cinco empresarios menonitas; se detectaron las características psicosociales de liderazgo empresarial y se determinó la diferencia entre los dos grupos empresarios según su cultura.

Resultados: En la región de Cuauhtémoc, Chihuahua, convergen dos culturas dominantes, una cultura mestiza y la otra cultura menonita, esta última, pertenece a un grupo religioso que por sus costumbres, ha desarrollado un estilo de vida característico. Se analizó la información y se encontró que existen diferencias significativas entre los dos grupos de empresarios entrevistados.
Gutiérrez-Enriquez E.C., Gutiérrez-Enriquez K.I. Culture as a determinant of business leadership in Ciudad Cuauhtémoc, Chihuahua. Hitos de Ciencias Económico Administrativas 2014;20 (58): 103-111.

\section{ABSTRACT}

Objective: To establish the cultural differences in business leadership, among employers of Cuauhtémoc, Chihuahua.

Material and method: The study was carried out in Ciudad Cuauhtémoc, Chihuahua., Personal information of individual entrepreneurs, was obtained by historybased entrepreneurial life entrepreneurs twelve men in the region of Cuauhtémoc, seven interviews were mestizos entrepreneurs five Mennonite entrepreneurs; psychosocial characteristics of entrepreneurial leadership were detected and the difference was determined between the two business groups according to their culture.

Results: In the region of Cuauhtémoc, Chihuahua., Two dominant cultures converge, a mestizo culture and other Mennonite culture, the latter belongs to a religious group for their customs, has developed a lifestyle characteristic. The information was analyzed and found that there are significant differences between the two groups of entrepreneurs interview.

* Profesora-Investigadora. Instituto Tecnologico de ciudad Cuauhtémoc.

** Docente. Instituto Tecnológico de Chihuahua. 
Conclusiones: Se concluye que los empresarios de la región de Cuauhtémoc, cuentan con características culturales específicas, que les han llevado a establecer un estilo de liderazgo empresarial, que les ha conducido a tener éxito como empresarios.
Conclusions: We conclude that entrepreneurs in the region of Cuauhtémoc, have specific cultural characteristics that have led them to establish a style of business leadership, which has led them to succeed as entrepreneurs.
Palabras clave: Diferencias culturales en el liderazgo empresarial. Características psicosociales. Empresarios de éxito.
Key words: Cultural differences in business leadership. Psychosocial characteristics.Ssuccessful

entrepreneurs.

DIRECCIÓN PARA RECIBIR CORRESPONDENCIA: Correo electrónico: cristigutie@gmail.com

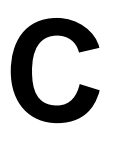

iudad Cuauhtémoc, Chihuahua, y su región, contemplan en la conformación de sus empresas principales y emergentes, una composición cultural de empresarios y trabajadores tanto menonitas como mestizos y una amplia planta de obreros provenientes de la Sierra Tarahumara. Dada esta estructura multicultural, el liderazgo empresarial, la estructura de grupo, la capacidad innovativa y la resistencia al cambio por parte de las organizaciones y sus empresarios, están en función de las diferencias individuales que presentan cada uno de los empresarios de los grupos étnicos que interactúan entre sí, lo cual genera diferencia en la visión de los objetivos y métodos para llegar a ellos, teniendo divergencias en cuanto al logro de los resultados y un eficiente desarrollo organizacional.

Así mismo, la función de dirigir la organización, va mucho más allá de la simple supervisión de que el trabajador cumpla con las actividades encomendadas, sino que cualquier persona que dirija y tenga a su cargo la responsabilidad de los resultados grupales, debe considerarse como un agente importante del cambio. De esta manera la efectividad de la función directiva, requiere de conocer cuales son los elementos psicosociales que caracterizan a la organización, sus dirigentes y su personal. Por la condición empresarial que prevalece en la región, se torna importante el determinar que características psicológicas y sociales han diferenciado a los empresarios de la región, y los han llevado a lograr consolidarse como líderes empresariales de éxito.

Desde que llegaron los primeros pobladores de la región de Cuauhtémoc, tanto hombres como mujeres, mostraron tener una gran calidad humana, inteligencia, capacidad de trabajar y entusiasmo, ya que la necesidad de sacar adelante a sus familias, los llevó a desarrollar una forma empresarial adecuada a las circunstancias, puesto que empezaron a trabajar la tierra y a comercializar sus productos, lo cual dio origen al surgimiento de empresas de comercialización y posteriormente de industrialización, de los productos del campo cómo semillas, fruta y derivados de la leche.

Los hechos históricos dan una idea de lo que la población de Cuauhtémoc ha desarrollado en los ámbitos social y económico, de tal forma que el crecimiento poblacional, muestra un cuantioso aumento en número de pobladores y de empresas, actualmente el municipio, a través de Fomento Económico (2013), tiene un registro mayor a 5050 empresas, algunas de ellas con una larga trayectoria empresarial; lo que da pie a considerar que en la Región de Cuauhtémoc, existen empresas y empresarios hombres y mujeres exitosos que mediante su formación cultural, técnica y estrategias empresariales utilizadas para dirigir les ha permitido mantenerse al frente de la organización y han sobrevivido en el mercado.

\section{MARCO TEÓRICO CONCEPTUAL}

La autora Gutiérrez (2009), cita que la aplicación de la psicología en las organizaciones se deriva según Davis y Newstrom (1995, 2003), de la importancia que el recurso humano tiene para éstas, las cuales buscan a través de dicha ciencia desarrollar las capacidades humanas al máximo para alcanzar niveles más elevados de competencia y creatividad organizacional. El estudio del comportamiento organizacional es relativamente nuevo, prácticamente tiene un siglo de 
estar realizándose, mientras que las organizaciones son bastante antiguas. En las empresas, se ha considerado la estructura básica que considera dos niveles jerárquicos, uno de jefe o patrón y otro de los trabajadores o subordinados, que hablan de una relación de poder entre las personas.

El comportamiento organizacional, se define según Gutiérrez (2005), como el estudio y análisis de la conducta que el factor humano desarrolla dentro de la organización, provocada por sus relaciones interpersonales, en un ambiente tanto físico como psicológico, que se ve afectado por el entorno social, económico y laboral que prevalece al momento de presentarse dicha conducta. Para Gutiérrez (2009), el comportamiento organizacional contempla el cambio de enfoque social que se ha manejado durante muchos años, trazando en los empleados una mentalidad de superación, con el enfoque de calidad total.

Alcover y Cols, (2004), dice que la evolución de la psicología diferencial, ha permitido el establecimiento de la medición de las diferencias individuales para su aplicación en el ámbito laboral. A este respecto, Hellriegel y Cols. (1999), señalan que es de suma importancia entender el comportamiento individual de las personas, en ambientes complejos como la organización, ya que el entorno y la influencia de las relaciones personales, generan comportamientos específicos. Robbins (2004), menciona que desde el punto de vista de la administración, lo importante no es establecer las diferencias individuales, sino saber que hace que la gente tenga éxito como empleado o como alguien que está al frente del grupo o empresa.

Los diferentes teóricos del comportamiento organizacional, coinciden en que son varios los factores que establecen las diferencias individuales, que permiten al individuo destacar en diferentes ámbitos sociales y laborales, estos factores oscilan desde los aspectos más complejos como la inteligencia, la personalidad, la cuestión de genética, hasta los aspectos menos complicados como la edad, estado civil, escolaridad. Sin embargo el hecho de que estos factores se conjuguen, da lugar a las características individuales que hacen a cada persona diferente lo que le permite considerarse como alguien exitoso o no.
El autor Chiavenato (1993), considera que la empresa «es una organización social que utiliza una gran variedad de recursos para alcanzar determinados objetivos", mientras que (Thompson, 2006), expone el concepto de empresa donde dice que «la empresa es una organización social que realiza un conjunto de actividades y utiliza una gran variedad de recursos (financieros, materiales, tecnológicos y humanos) para lograr determinados objetivos, como la satisfacción de una necesidad o deseo de su mercado meta con la finalidad de lucrar o no; y que es construida a partir de conversaciones específicas basadas en compromisos mutuos entre las personas que la conforman».

El Diccionario de la Lengua Española (2005), define empresario como «la persona que posee o dirige una industria, negocio o empresa», mientras que la Organización de las Naciones Unidas para la Agricultura y la Alimentación (FAO), (2008), define empresario como «la persona o empresa que desea emprender una actividad mercantil, reuniendo los recursos necesarios y arriesgándose al éxito o fracaso». De igual forma, El Ministerio de Comercio, Industria y Turismo de Colombia, a través de su portal empresarial (2006), definen al empresario como «un emprendedor que asume el reto de crear una empresa, el empresario es un protagonista de la vida económica que con su esfuerzo, disciplina y dedicación logra mantener vigente su empresa, de hacerla competitiva, de vencer adversidades, de aprender y desaprender día a día».

Para que una empresa $u$ organización pueda ser considerada del Siglo XXI, Mora (2005), argumenta que deberá cumplir con las siguientes características: estar orientada al conocimiento, contar con personal altamente calificado, desarrollar el potencial creativo e innovador de su personal, orientarse a la satisfacción del cliente, tener una gran capacidad de aprender y promover una estructura plana y flexible.

Las cualidades personales para liderar una organización con éxito, según Daft (2006), pueden ser natas o desarrolladas con el tiempo, y el autor distingue cuatro de ellas específicamente, la primera, tener una visión personal de largo alcance que reconozca y apoye una comunidad organizacional diversa, la segunda, tener un amplio conocimiento de las dimensiones de la diversidad y una conciencia de las cuestiones multiculturales, la 
tercera, tener disposición para cambiar ellos mismos; y por último, guiar a los empleados y enseñarles a tomar decisiones, delegando autoridad y responsabilidad.

Argumenta Holland (2008), quién es fundador de una compañía prestigiosa en Estados Unidos, Yum, Yum, Donut Shop Inc., que las características que debe tener un empresario para considerarse de éxito, son los siguientes aspectos, como ser autosuficiente, tener ganas y disfrutar de la operación de una empresa, ser el mejor en el producto que fabrica o servicio que se brinda, de tal forma que se convierta en especialista de su ramo, concentrarse en la satisfacción del cliente, tener la experiencia directa y personal de estar al frente del negocio y por último tener conocimientos básicos de contabilidad y de flujo de fondos.

El empresario nace con algunas de las características que lo pueden hacer alcanzar el éxito, pero, puede desarrollar otras características que también le serán de utilidad, según lo señala Guerrero (2005), y argumenta que estas son las características que diferencian a los empresarios de éxito de los demás empresarios: fijación continua de objetivos, perseverancia, conocimiento del negocio, tolerancia al fracaso, autonomía, iniciativa, tomar riesgos, son persistentes en la solución de problemas, empuje, conciencia de capacidades y límites, salud física y mental, manejar la incertidumbre, autocompetencia, responsabilidad, confianza en sí mismo, versatilidad, ser realista, positivo, ser flexible, innovador, creativo, tiene trabajadores a largo plazo, planea a largo plazo, constante, capacidad de relacionarse, de vender la idea, apoyo de la familia, educación, y tener la edad entre treinta y treinta y cinco o entre cincuenta y cincuenta y cinco años.

Según el estudio desarrollado por la Cátedra PERI - IE de Liderazgo Empresarial en el (2006), «los líderes representan la visión de futuro de la empresa, pero esta visión está influenciada por los compañeros de trabajo. Este estudio sugiere, que, en la implementación del cambio y la innovación, los nuevos líderes tienen que ir más allá de la autoridad formal para gestionar además las redes informales de comunicación». Además, los autores del estudio aseguran que las condiciones económicas y sociales tan difíciles y cambiantes, junto con la globalización y la exigencia de conocimiento, están obligando a los líderes empresariales a ir más allá de la armonía de personas y acciones del momento, sino que deberán desarrollar una cultura de cambio y diferentes estilos de liderazgo basados en la innovación y responsabilidad social hacia la empresa, su gente y el entorno.

El teórico Giraldo (2008), cita a varios autores como Carrión (1998), quién define el liderazgo empresarial como la función ejercida por dirigentes empresariales que tienen interés de influir no solo en su empresa, sino también en el ámbito social, político y económico, a través de agrupaciones empresariales y gubernamentales, así mismo cita a Gupta y MacMillan (2004), quienes sugieren que los líderes empresariales se enfrentan a dos desafíos, el primero enfocado a la creación de condiciones adecuadas para desarrollar una empresa con los recursos disponibles y el segundo se refiere a la necesidad de convencer a la gente de coordinar esfuerzos para aprovechar los recursos y alcanzar los objetivos. Los autores señalan que el líder empresarial desempeña cinco acciones principalmente: Planear y generar el cambio, minimizar la incertidumbre y la resistencia al cambio, generar compromisos y por último especificar los límites.

Presentan Landy y Conte (2005), un análisis de las diferencias entre el liderazgo masculino y femenino en un escenario organizacional, considerando que la industria particular está dominada por hombres o mujeres; por lo que citan el estudio hecho por Gardiner y Tiggeman (1999), quienes analizaron entre sesenta hombres y sesenta mujeres gerentes de industrias de dominio masculino, como son industrias, organizaciones de automotores, tecnología de la información y de transformación de madera entre otras, y también organizaciones de dominio femenino, como serían educación infantil, estéticas y enfermería, entre otras. Encontraron que los escenarios de dominio masculino, no favorecen el estilo de liderazgo femenino orientado a las relaciones interpersonales, y si las gerentes se esforzaban por mantener una orientación hacia la relación, su salud mental se mermaba, contrario a los gerentes hombres que adoptaban un estilo orientado a la relación, en este mismo escenario, los cuales mejoraron su salud mental. Por lo que se concluyó que las mujeres al tratar de mantener su forma esperada 
de actuación, encontraron resistencia, lo cual no sucedió con los hombres al actuar en forma no esperada.

La investigadora Stelter (2002), citada por Daft (2006), señala que en las investigaciones hechas para identificar la forma de actuar de los líderes hombres y mujeres, se ha encontrado que los hombres actúan en forma agresiva y seguros de sí mismo, mientras que las líderes mujeres, se identifican por su interés en las relaciones, en la inclusión de la gente en el grupo, en la participación y el cuidado de otros. Una líder como Deborah Kent, quien fue la primera mujer en dirigir una planta de montaje de vehículos, para Ford Motor Co., se caracterizó por estar dispuesta a compartir el poder y la información, a estimular el desarrollo de los trabajadores y a luchar por mantener el valor personal de los demás.

\section{METODOLOGÍA}

La presente investigación es de tipo cualitativa, en la cual se analizó e interpretó la información proporcionada por los empresarios, mediante entrevistas, con el objetivo de determinar las diferencias culturales en las características de liderazgo empresarial. Se consideró una muestra de doce empresarios que están al frente de las empresas, pertenecientes a dos grupos étnicos, siete de ellos son mestizos y cinco son menonitas de la región de Ciudad Cuauhtémoc.

El instrumento de recopilación de la información que se utilizó fue, la entrevista a profundidad, con cada uno de los empresarios, con preguntas abiertas en torno a la vida empresarial de cada uno de ellos, en base a los siguientes aspectos: historia personal, historia de la empresa, visión que cada uno tiene de su empresa, expectativa al iniciar el negocio, estrategias utilizadas para dirigir, etapas más significativas en su trayectoria, etapa actual como empresario, aspectos personales, participación de la familia en la empresa y expectativas a futuro.

\section{RESULTADOS}

El análisis de la información proporcionada por los siete empresarios mestizos entrevistados, y los cinco empresarios menonitas, arrojó los siguientes resultados:
Las características de liderazgo empresarial predominantes en los empresarios mestizos, esta enfocada a un estilo de liderazgo transaccional, en su mayoría, ya que procuran no endeudarse, reconocen el desempeño de sus trabajadores, los tratan bien y se preocupan por su bienestar, buscan pertenecer a grupos empresariales, buscan dar buen servicio al cliente, coinciden en que tenían un conocimiento previo del negocio al iniciarlo, se visualizaron como No. 1 en su ramo, dos de las empresas tienen reconocimiento a nivel nacional por su calidad, los empresarios se consideran cautelosos, precavidos, organizados, constantes, agradecidos, responsables, honestos, disciplinados, metódicos, están conscientes de sus capacidades y límites, siguen su instinto, implementan cambios paulatinamente, fomentan las relaciones armoniosas y se mantienen con ideas y decisiones firmes (ver tablas 1 y 2 ).

En los empresarios del grupo cultural menonita predominan las siguientes características se visualiza una tendencia de liderazgo participativo, ya que buscan tomar decisiones en familia, así como mantener una relación estrecha con su personal, consideran que la educación académica es muy importante para tener éxito, su empresa está constituida en sociedad, procuran delegar autoridad, toma de decisión y responsabilidad, buscan el crecimiento de la empresa paso a paso, se informa sobre condiciones del mercado y se adaptan a ellas, dos de los empresarios son ministros de la iglesia y todos ellos agradecen a Dios, por su empresa. Los cinco empresarios expresaron que iniciaron por la necesidad económica y se consideran arriesgados y tenaces, algunos se visualizaron con empresa propia, además la mayoría de ellos se sienten confiados en su capacidad, se consideran positivos, creativos, entusiastas, empeñosos, activos, persistentes les gusta actualizarse asistiendo a congresos, trabajar en equipo y se visualizan como líder (ver tablas 1 y 2.)

Dentro del análisis se encontró que las características que tienen en común los dos grupos de empresarios son el trabajar con la familia, procuran reinvertir en el negocio, establecen cambios de producto o giro, se enfocan en reducir gastos y optimizar los recursos, así como en dar calidad en el servicio y/o producto, analizan el mercado, tratan de perseguir su objetivo de diferentes 
formas, se consideran analíticos, innovadores, respetuosos, trabajadores, perseverantes, luchistas, se esfuerzan, tienen amor por la empresa o profesión y hacen planes.

\section{CONCLUSIONES}

Afín a lo que señala Gutiérrez (2005), que existen diferencias individuales que caracterizan a cada persona, ya que se encontraron características diferentes en los dos grupos de empresarios. Así mismo, Alcover y Cols. (2004), dice que la evolución de la psicología diferencial, ha permitido el establecimiento de la medición de las diferencias individuales para su aplicación en el ámbito laboral. A este respecto, Hellriegel y Cols. (1999), señalan que es de suma importancia entender el comportamiento individual de las personas, en ambientes complejos como la organización, ya que el entorno y la influencia de las relaciones personales, generan comportamientos específicos. Robbins (2004),

\section{Tabla 1}

Características psicológicas con mayor incidencia entre los empresarios

\begin{tabular}{|c|c|c|}
\hline Característica Psicológica & $\begin{array}{c}\text { Empresarios } \\
\text { M estizos }\end{array}$ & $\begin{array}{c}\text { Empresarios } \\
\text { Menonitas }\end{array}$ \\
\hline Cauteloso, precavido & 4 & \\
\hline Implementa cambios paulatinos & 4 & \\
\hline Persigue el objetivo & 7 & 5 \\
\hline Sigue su instinto & 4 & \\
\hline Organizado, ordenado & 6 & \\
\hline Fomenta relaciones armoniosas & 5 & \\
\hline $\begin{array}{l}\text { Está consciente de sus capacidades y } \\
\text { límites }\end{array}$ & 6 & \\
\hline Constante & 4 & \\
\hline Ideas y decisiones firmes & 4 & \\
\hline Innovador & 4 & \\
\hline Respetuoso & 7 & 5 \\
\hline Trabajador & 5 & 5 \\
\hline Perseverante, luchista & 5 & 5 \\
\hline Amor por la empresa, profesión o trabajo & 4 & 4 \\
\hline $\begin{array}{l}\text { Busca diferentes formas de alcanzar el } \\
\text { objetivo }\end{array}$ & 4 & 5 \\
\hline Planea & 4 & 3 \\
\hline Responsable & 5 & \\
\hline Honesto, honrado & 4 & \\
\hline Inició por necesidad económica & & 5 \\
\hline Visión: tener una empresa propia & & 3 \\
\hline Confianza y seguridad en sí mismo & & 3 \\
\hline $\begin{array}{l}\text { Esta consciente de sus capacidades y } \\
\text { límites }\end{array}$ & & 3 \\
\hline Positivo & & 4 \\
\hline Creativo & & 4 \\
\hline Innovador & & 4 \\
\hline Entusiasta, empeñoso, activo & & 4 \\
\hline Persistente & & 3 \\
\hline Arriesgado y tenaz & & 5 \\
\hline
\end{tabular}

Fuente: elaboración propia. 


\section{Tabla 2}

\section{Característcas sociales con mayor incidencia entre los empresarios.}

\begin{tabular}{lcc}
\hline \multicolumn{1}{c}{ Característica Social } & $\begin{array}{c}\text { Empresarios } \\
\text { Mestizos }\end{array}$ & $\begin{array}{c}\text { Empresarios } \\
\text { Menonitas }\end{array}$ \\
\hline No endeudarse & 4 & 4 \\
Reinvertir & 7 & \\
Reconoce el desempeño de los trabajadores & 4 & \\
Pertenece a grupos de empresarios & 5 & 3 \\
Da buen servicio al cliente & 5 & \\
Promueve productos nuevos u otro giro & 4 & 4 \\
Tenía conocimiento del negocio al iniciarlo & 4 & 4 \\
Trata muy bien a sus empleados & 4 & 4 \\
Reduce gastos, optimiza recursos & 5 & 3 \\
Calidad en el servicio y/o producto & 4 & 4 \\
Trabaja con la familia & 5 & 3 \\
Asiste a congresos & 1 & 4 \\
Cree que la educación académica lleva al éxito & & 5 \\
Mantiene contacto estrecho con su personal & & 4 \\
La empresa es una sociedad mercantil & & \\
Agradece a Dios & & \\
Se adapta a las condiciones del mercado &
\end{tabular}

Fuente: elaboración propia.

menciona que desde el punto de vista de la administración, lo importante no es establecer las diferencias individuales, sino saber que hace que la gente tenga éxito como empleado o como alguien que está al frente del grupo o empresa; por tanto, los resultados encontrados, están acordes a lo señalado anteriormente por los teóricos.

Del total de las características encontradas, hay una característica que está en el cien por ciento de ellos, es el respeto valor social básico para el desarrollo armonioso de una comunidad y en este caso de una empresa, característica mencionada por Guerrero (2005).

De las características que se presentan en la mayoría de los empresarios mestizos, se observan cuatro características que están en seis o siete de ellos, estas son: perseguir el objetivo y ser respetuoso que están en todos los entrevistados y ser organizado, ordenado y estar consciente de sus capacidades y límites, que se encuentran en seis de ellos, por lo que puede decirse que son las características distintivas de ese grupo de empresarios. Lo cual muestra una diversidad entre ellos de formas de pensar y actuar, viendo que la cultura donde se mueven, genera diferencias individuales notorias, sin embargo, aunque ellos sean diferentes entre sí, todos han alcanzado el éxito empresarial de una forma o de otra.

Por el contrario, al grupo anterior de empresarios, el grupo de menonitas, presenta las características comunes entre ellos, once que están en cuatro o cinco de ellos, como haber iniciado por necesidad económica, perseguir el objetivo, ser respetuoso, trabajador, creativo, innovador, entusiasta y tener amor por lo que hacen. Esto muestra, que el ambiente socio cultural donde se desarrollan estos empresarios, la forma en que se educan en familia y su forma de vida en general, promueve y facilita comportamientos similares entre sus habitantes, por lo que las diferencias de actuación entre ellos son menos, sin embargo, de igual forma estos empresarios, han alcanzado el éxito. 
En el análisis comparativo entre el grupo de empresarios mestizos y el grupo de empresarios menonitas, se observa que tienen algunas de características en común los dos grupos, estás son nueve características, entre las cuales destacan ser respetuoso, perseguir el objetivo, ser trabajador, perseverante, luchista, tener amor por lo que hacen, hacer planes y buscar diferentes formas de alcanzar el objetivo, entre otras, esto indica que por género masculino, existe mayor afinidad en la forma de actuar.

De manera general, se puede concluir que la región de Cuauhtémoc Chihuahua, cuenta orgullosamente con empresas y empresarios de éxito, que han demostrado tener las características psicológicas y sociales necesarias para mantener en el mercado sus empresas por más de treinta años como lo han hecho la mayoría de ellos.

El estudio está lleno de información, emociones y experiencias que hacen sentir una gran satisfacción, por tener la oportunidad de que estos empresarios contribuyan al desarrollo económico y social de la comunidad.

Por último, cabe una recomendación para los empresarios jóvenes o novatos en el ramo empresarial, acérquense a aquellas personas que tienen toda una vida haciéndolo, aprendan de ellos, que por lo que ya se vio, cuentan con algunas características que tenían al nacer, pero otras de ellas las tuvieron que aprender a través de su trayectoria por la vida les permitió a base de esfuerzo, dedicación, paciencia y perseverancia, superar los tropiezos del andar cotidiano, y llegar hasta el nivel en el que actualmente se encuentran sus empresas, consideradas todas ellas como «EXITOSAS».

\section{REFERENCIAS}

Alcover de la Hera C., Martínes D., Rodríguez F., y Domínguez R. (2004). Introducción a la Psicología del Trabajo. España: McGrawHill.

Chiavenato I. (1993). Administración de Recursos Humanos Edición: 1era. México: Mc Graw-Hill.

Daft R. (2006). La Experiencia del Liderazgo tercera edición. México: Thomson Editorial

Diccionario de la Lengua Española, (2005). Madrid: Espasa-Calpe S.A.

Food and Agriculture Organization of the United Nations, (2008). Recuperado de www.FAO.org

Giraldo T. (2008). Responsabilidad Social, Sustentabilidad y Liderazgo Empresarial en Colombia: un Análisis Intersectorial. Colegio de Estudios Superiores de Administración-CESA. Colombia.

Guerrero. (2005). Principales Características de los Empresarios de Éxito. Chile: Universidad Tecnológica Intercontinental.

Gutiérrez, E. (2005). Formación de la Conducta Organizacional. Instituto Tecnológico de Cd. Cuauhtémoc, México.

Gutiérrez E. (2009). Descripción de las características psicosociales de liderazgo de los empresarios exitosos de la Región de Cuauhtémoc. Instituto Tecnológico de Ciudad Cuauhtémoc, Chihuahua. México.

Hellriegel D., Slocum J., Woodman R. (1999). Comportamiento Organizacional. México. Thompson Editores. 
Holland P. (2008). De modo que usted quiere tener su propia pequeña empresa. Departamento de Estado de Estados Unidos. Estados Unidos. Oficina de Programas de Información Internacional.

Landy F., y Conte J. (2005). Psicología Industrial. México: McGrawHill

Mipymes Portal Empresarial Colombiano (2006). Ministerio de Comercio, Industria y Turism. Recuperado de www.mipymes.gov.co

Mora C. (2005). Organizaciones Exitosas del Siglo XXI. Área de estudios de Postgrado de la Universidad de Carabobo. Venezuela.

Recuperado de www.cmoraarrobapostgrado.uc.edu.ve \& www. camv12arrobahotmail.com

Robbins, S. (2004). Comportamiento

Organizacional. México: Editorial Prentice Hall

Recuperado de www.utic.edu.py/bdigital/biblio/ index.php?dir=EMPRESARIALES/

EMPRENDEDOR/\&file...doc

Thompson I. (2006). Definición de Empresa. Promonegocios.net. Portal de mercadotecnia. México: Recuperado de www.promonegocios.net 